\title{
Rotavirus $G$ and P Types Circulating in the Eastern Region of Kenya
}

\author{
Predominance of G9 and Emergence of G12 Genotypes
}

\author{
Nicholas M. Kiulia, MSc, * Martin M. Nyaga, MSc, † Mapaseka L. Seheri, PhD, † Marianne Wolfaardt, PhD, $\neq$ \\ Walda B. van Zyl, PhD, \$ Mathew D. Esona, PhD, § Grace Irimu, PhD, \ Murithi Inoti, Mmed,\|l \\ Bernard W. Gatinu, Mmed, ** Peter K. Njenga, PhD, †† Maureen B. Taylor, DSc $+\neq \neq$, and Atunga Nyachieo, PhD*
}

\begin{abstract}
Background: The World Health Organization has recommended that rotavirus (RV) vaccines be included in all national immunization programs as part of a strategy to control RV-associated diarrheal diseases. Hospitalbased surveillance of RV infection is therefore crucial in monitoring the impact pre- and post-vaccine introduction and also to document changes in genotype distribution. This study sought to determine the RV genotypes circulating in the eastern region of Kenya before introduction of the RV vaccine.
\end{abstract}

Methods: During September 2009 to August 2011, 500 stool samples were collected from children $<5$ years of age admitted for acute diarrhea in hospitals in the eastern region of Kenya and analyzed for the presence of group A RV using an enzyme immunoassay. G and P genotypes were determined using hemi-nested reverse transcriptase polymerase chain reaction.

Results: One hundred and eighty nine out of 500 (38\%) samples analyzed were positive for rotavirus. The following $\mathrm{G}$ types were detected: G9 (50.9\%), G1 (26.8\%), G8 (12.1\%), G12 (3.1\%), G2 (0.6\%), mixed G (1.3\%) and $5.1 \%$ were $\mathrm{G}$ nontypeable. $\mathrm{P}$ types detected included: $\mathrm{P}[8](63.7 \%)$, $\mathrm{P}[4]$ (12.1\%), P $[6]$ (4.5\%), mixed P (7.6\%) and $12.1 \%$ were P nontypeable. The most dominant strain was G9P[8] (35\%), followed by G1P[8] (26.8\%), G8P[4] (9.6\%), G12P[6] (2.5\%), G9P[6] (1.9\%), G9P[4] (1.3\%), G8P[8] (1.3\%), and G2P[4] (0.6\%).

Conclusions: The present study demonstrates the recurring changing genotypes of RV circulating in Kenya, with genotypes G9, G1 and G8 being the dominant strains circulating in the eastern region of Kenya between 2009 and 2011. Additionally, G12 genotype was detected for the first time in Kenya.

Key words: rotavirus, gastroenteritis, genotypes, vaccines

(Pediatr Infect Dis J 2014;33:S85-S88)

Accepted for publication August 27, 2013.

From the *Enteric Viruses Research Group, Institute of Primate Research, Karen, Nairobi, Kenya; $†$ Regional Rotavirus Reference Laboratory, MRC/Medunsa Diarrheal Pathogens Research Unit, University of Limpopo; $\$$ Department of Medical Virology, Faculty of Health Sciences, University of Pretoria, Pretoria, South Africa; §Gastroenteritis and Respiratory Viruses Laboratory Branch, Centers for Disease Control and Prevention, Atlanta, GA; - Department of Paediatrics, School of Medicine, University of Nairobi, Nairobi; IMaua Methodist Hospital, Maua; **Meru General Hospital, Meru; ††Department of Applied Science, Medical Microbiology Program, Jomo Kenyatta University of Agriculture and Technology Karen Campus, Nairobi, Kenya; and $\$$ National Health Laboratory Service, Tshwane Academic Division, Pretoria, South Africa.

N.M.K., M.B.T. and A.N. have received funding from Merck \& Co., Inc., NJ, for RV disease surveillance studies. The authors have no other funding or conflicts of interest to disclose.

The findings and conclusions in this article are those of the authors and do not necessarily represent the views of Merck.

Disclaimer: The findings and conclusions in this report are those of the authors and do not necessarily represent the views of the CDC.

Address for Correspondence: Nicholas M. Kiulia, Enteric Viruses Research Group, Institute of Primate Research, PO. Box 24481-00502, Karen, Nairobi, Kenya. E-mail: nick.kiulia@gmail.com.

Copyright (C) 2013 by Lippincott Williams \& Wilkins

ISSN: 0891-3668/14/3301-0S85

DOI: $10.1097 / \mathrm{INF} .0000000000000059$
Croup A rotaviruses ( $\mathrm{RVs}$ ) remain the major cause of morbidity and mortality globally and are associated with an estimated 453,000 deaths (range 420,000-494,000 deaths) annually in infants and young children $<5$ years of age, ${ }^{1}$ and approximately $85 \%$ of these deaths occurring in Sub-Saharan Africa. ${ }^{1,2}$ Infants and young children $<2$ years of age are most vulnerable to RV infection that often results in severe diarrhea and dehydration, causing hospitalization which can result in death. ${ }^{3}$ This age group has been the primary target of protection with RV vaccines, and large-scale efficacy and safety trials of 2 RV vaccines, Rotarix (GlaxoSmithKline, Rixensart, Belgium), containing a single human G1P[8] RV strain, and RotaTeq (Merck \& Co., Whitehouse Station, NJ), containing 5 bovine-human reassortant RV strains expressing 5 human serotypes (G1, G2, G3, G4 and P[8]) have demonstrated the impact of these vaccines in developing countries. ${ }^{4-8}$ The recent clinical trials in Africa and Asia ${ }^{7-9}$ found that the vaccine significantly reduces severe diarrhea episodes due to RV by $48-64 \%$ during the first year of life. These vaccines have been reported to induce significant protection against severe diarrhea caused by homotypic and heterotypic RV strains ${ }^{4,5,10,11}$ and reduce childhood deaths. ${ }^{12}$ The Rotarix and RotaTeq RV vaccines have proved to have high efficacy in developed countries. ${ }^{4,6,13}$ Available since 2006, these vaccines have been licensed in many countries including Kenya. ${ }^{14,15}$

The recommendation by World Health Organization (WHO) that RV vaccines be included in all national immunization programs, particularly in those countries where diarrheal deaths account for $>10 \%$ of $<5$ mortality, ${ }^{16}$ has resulted in many countries applying for financial support from Global Alliance for Vaccines and Immunization to enable introduction of these vaccines. Based on WHO recommendations, Global Alliance for Vaccines and Immunization and other international partners have agreed to provide financial support for RV immunization in the developing world and the WHO Regional Office for Africa has been spearheading the sentinel surveillance of RV for the last 6 years. ${ }^{15}$ The main aim of the ongoing RV hospitalbased surveillance studies being conducted in Africa and coordinated by the Regional Office for Africa of WHO is to monitor the burden of $\mathrm{RV}$ infection, prevalence of predominant genotypes and identify unusual and emerging strains in the continent. ${ }^{15}$ Such studies are particularly important in countries such as Kenya that are considering introducing the RV vaccine in the expanded program for immunization.

$\mathrm{RV}$ is a non-enveloped virus with a triple-layered capsid containing 11 double-stranded RNA segments surrounded by 3 concentric protein layers. The outer capsid consists of VP7 (a glycoprotein) and VP4 (a protease-sensitive protein) which carry independent neutralization and protective antigens. ${ }^{17} \mathrm{~A}$ binary system of RV classification has been established to designate the neutralization specificity of the VP7 and VP4 proteins. The VP7 genotype is known as G and the VP4 genotype as $\mathrm{P}^{17}$ To date, 27 different $\mathrm{G}$ and $35 \mathrm{P}$ genotypes have been described in both humans and animals. ${ }^{18}$ Globally, human infections have been mainly caused by 5 G types (G1-G4 and G9) and $3 \mathrm{P}$ types $(\mathrm{P}[4], \mathrm{P}[6]$ and $\mathrm{P}[8]) \cdot{ }^{17,19,20}$ Epidemiological surveillance of circulating RV strains is critical in developing countries to 
determine the protective efficacy of RV vaccines against multiple genotypes and to detect the eventual emergence of different strains. In Kenya, epidemiological studies have been done to determine the circulating RV genotypes and have showed the clinical importance of RV disease in young Kenyan children. ${ }^{15,21-24}$ Before the introduction and implementation of a universal RV vaccination program, continued monitoring of RV strain variations is important so that the impact of vaccination and any subsequent changes in circulating strains can be determined.

The main aim of this study was to determine the distribution of circulating $\mathrm{G}$ and $\mathrm{P}$ genotype among children $<5$ years of age admitted to hospitals with acute gastroenteritis in the eastern region of Kenya before RV vaccination introduction.

\section{MATERIAL AND METHODS}

\section{Study Design}

This study was a prospective hospital-based surveillance study in the eastern region of Kenya. The study was carried out in Meru General Hospital and Maua Methodist Hospital in Meru County. These hospitals are in close vicinity approximately $50 \mathrm{~km}$ apart and they both serve the same catchment area.

\section{Collection of Stool Specimens}

During September 2009 to August 2011, 500 fecal samples were collected from children $<5$ years of age admitted with acute diarrhea in Meru General Hospital and Maua Methodist Hospital in the eastern region of Kenya. These hospitals were selected based on the earlier study by Kiulia et al $(2006)^{21}$ and fulfilled requirements for RV surveillance study as outlined by WHO. ${ }^{15,25}$

Fecal samples and epidemiological data were collected from all children $<5$ years of age with severe nonbloody diarrhea admitted to the pediatrics ward and/or who visited the emergency department for treatment for acute gastroenteritis during the study period. A case of acute gastroenteritis was defined as the passage of $\geq 3$ looser than normal stools within a 24 -hour period or $\geq 2$ episodes of vomiting unexplained by other reason and as described by the guardian or mother at presentation to the hospital.

\section{RV Detection}

A $10 \%$ suspension of fresh stool in sample diluent was made and tested for the presence of group A RV antigen using a commercial RV antigen detection enzyme immunoassay (ProSpecT Rotavirus test, Oxoid, Ely, United Kingdom) according to the manufacturer's instructions.

\section{Viral RNA Extraction for Reverse Transcriptase Polymerase Chain Reaction}

RV dsRNA was extracted using the QIAamp Viral RNA kit (QIAGEN GmbH, Hilden, Germany) according to the manufacturer's instructions, with slight modification. Briefly, a $10 \%$ stool suspension was made in phosphate-buffered saline solution. The suspension was votexed, spinned down and supernatant used for RNA extraction. Purified extract was aliquoted and stored at $-20^{\circ} \mathrm{C}$ until use.

\section{VP7 and VP4 Genotyping of RV Strains}

The VP7 and VP4 reverse transcriptase polymerase chain reaction (RT-PCR) amplification and genotyping were carried out as described elsewhere. ${ }^{26-29}$

\section{Ethical Approval}

This study was approved by the Kenyatta National Hospital Ethics and Research Committee (Number: P257/09/2008), Institutional Review committee of the Institute of Primate Research (Number: IRC/01/2009) and Maua Methodist Hospital Research Committee
(MMH/08/2009). In addition, informed consent was obtained from each child's guardians/parents before enrolment into the study.

\section{RESULTS}

\section{Prevalence of RV in Eastern Region}

A total of 500 children with diarrhea were enrolled in the study with 189 (38\%) found to be positive for RV infection by enzyme immunoassay.

\section{Distribution of Circulating $\mathrm{G}$ and $\mathrm{P}$ Genotypes}

Of the 189 enzyme immunoassay positive samples, 157 were randomly selected for further characterization by RT-PCR and they were representative of samples collected during the study period. Both $G$ and $P$ types were detected in 137/157 (87.3\%) samples, while $7 / 157$ (4.5\%) samples remained nontypeable for both $G$ and $\mathrm{P}$ types (Table 1).

During the study period, the following $G$ types were detected: G9 (50.9\%), G1 (26.8\%), G8 (12.1\%), G12 (3.1\%) and $\mathrm{G} 2(0.6 \%)$. Mixed G types were also detected in $2(1.3 \%)$ samples, and no $\mathrm{G}$ types were obtained in 8 samples $(5.1 \%)$. The following P types were detected: $\mathrm{P}[8](63.7 \%), \mathrm{P}[4](12.1 \%)$ and $\mathrm{P}[6]$ (4.5\%). Mixed P type was found in $7.6 \%$ of samples and in an unexpectedly high percentage (12.1\%) of samples were P nontypeable. The most dominant genotype was G9P [8] (35\%), followed by G1P[8] (26.8\%), G8P[4] (9.6\%) and G12P[6] (2.6\%). Other G and P type combinations detected were G9P [6] (1.9\%), G9P[4] (1.3\%), G8P[8] (1.3\%) and G2P[4] (0.6\%; Table 1). Genotypes G3 and G4 were not detected in any of the samples analyzed during the study. The mixed $G$ types detected were mostly G9/G12 and mixed $P$ types were $\mathrm{P}[8]+\mathrm{P}[4]$ and $\mathrm{P}[8]+\mathrm{P}[6]$ (Table 1).

In this study, genotype G9 predominated throughout the entire period (Table 1). G1 was detected during the surveillance period 2010-2011 and G12 detected in both season 2009-2011. G2 genotype was only found during the surveillance period 2009-2010 and then at very low levels $(0.6 \%)$.

TABLE 1. Distribution of RV Strains Circulating Among Children Hospitalized With Acute Gastroenteritis in the Eastern Region, Kenya 2009-2011

\begin{tabular}{lccc}
\hline \hline & \multicolumn{2}{c}{ Year of Surveillance } & $\begin{array}{c}\text { Total } \\
\text { Frequency } \\
(\%)^{*}\end{array}$ \\
\cline { 2 - 3 } Genotype & $2009-2010$ & $2010-2011$ & $42(26.8)$ \\
G1P[8] & 0 & 42 & $1(0.6)$ \\
G2P[4] & 1 & 0 & $11(7.0)$ \\
G8P[4] & 6 & 5 & $2(1.3)$ \\
G8P[8] & 0 & 2 & $1(0.6)$ \\
G8P[NT] & 0 & 1 & $1(0.6)$ \\
G8P[4,8] & 1 & 0 & $2(1.3)$ \\
G9P[4] & 0 & 2 & $3(1.9)$ \\
G9P[6] & 1 & 2 & $55(35.0)$ \\
G9P[8] & 18 & 37 & $11(7.0)$ \\
G9P[NT] & 0 & 5 & $5(3.2)$ \\
G9P[4,8] & 0 & 1 & $4(2.5)$ \\
G9P[6,8] & 3 & 2 & $4(2.5)$ \\
G12P[6] & 2 & 1 & $1(0.6)$ \\
G12P[4,8] & 0 & 1 & $1(0.6)$ \\
G9,12P[8] & 0 & 1 & $1(0.6)$ \\
GNTP[4] & 0 & 2 & $7(4.5)$ \\
GNTP[NT] & 5 & 1 & $1(0.6)$ \\
G9/12P[4,8] & 0 & $120(76.4)$ & $157(100)$ \\
Total $(\%)$ & $37(23.6)$ & & \\
* The percentage with respect to the total number of positive in each genotype. &
\end{tabular}

NT, nontypeable. 
TABLE 2. Distribution G and P Genotype According to Age Categories

\begin{tabular}{|c|c|c|c|c|c|c|}
\hline \multirow[b]{2}{*}{ Genotypes } & \multicolumn{6}{|c|}{ Number of Strains, by Patient Age Group } \\
\hline & $0-6$ months & 7-12 months & 13-24 months & $25-36$ months & $37-48$ months & Tota \\
\hline G1P[8] & 26 & 16 & 0 & 0 & 0 & 42 \\
\hline $\mathrm{G} 2 \mathrm{P}[4]$ & 0 & 1 & 0 & 0 & 0 & 1 \\
\hline G8P[4] & 6 & 3 & 2 & 0 & 0 & 11 \\
\hline G8P[8] & 0 & 2 & 0 & 0 & 0 & 2 \\
\hline G8P $[\mathrm{NT}]$ & 0 & 1 & 0 & 0 & 0 & 1 \\
\hline G8P $[4,8]$ & 0 & 1 & 0 & 0 & 0 & 1 \\
\hline G9P[4] & 2 & 0 & 0 & 0 & 0 & 2 \\
\hline G9P[6] & 1 & 1 & 0 & 1 & 0 & 3 \\
\hline G9P[8] & 31 & 21 & 3 & 0 & 0 & 55 \\
\hline G9P[?] & 3 & 5 & 3 & 0 & 0 & 11 \\
\hline G9P $[4,8]$ & 3 & 2 & 0 & 0 & 0 & 5 \\
\hline G9P $[6,8]$ & 3 & 1 & 0 & 0 & 0 & 4 \\
\hline $\mathrm{G} 12 \mathrm{P}[4]$ & 4 & 0 & 0 & 0 & 0 & 4 \\
\hline G12P[6] & 1 & 2 & 1 & 0 & 0 & 4 \\
\hline $\mathrm{G} 12 \mathrm{P}[4,8]$ & 0 & 1 & 0 & 0 & 0 & 1 \\
\hline G9,12P[8] & 0 & 1 & 0 & 0 & 0 & 1 \\
\hline GNTP[4] & 1 & 0 & 0 & 0 & 0 & 1 \\
\hline GNTP[NT] & 2 & 2 & 0 & 0 & 3 & 7 \\
\hline G9/12P $[4,8]$ & 1 & 0 & 0 & 0 & 0 & 1 \\
\hline Total & 84 & 60 & 9 & 1 & 3 & 157 \\
\hline
\end{tabular}

NT, nontypeable.

\section{Age Distribution and G and P Genotypes}

The age distribution of patients to the RV genotypes circulating in the eastern region of Kenya is shown in Table 2. RV infection was found in all the age groups at different frequencies. Among the globally common strains, G9P[8] and G8P[4] was found circulating in all age groups except in older children $>25$ months of age, whereas $\mathrm{G} 1 \mathrm{P}[8]$ and G2P[4] were identified in younger children (7-12 months of age). G12P[6] were found circulating among children $<24$ months of age. Unusual strains and mixed infections were also found circulating in different age groups although at lower levels.

\section{DISCUSSION}

This study was performed to provide data on the circulating $\mathrm{RV}$ genotypes in the eastern region of Kenya, in anticipation of possible introduction of RV vaccine in the expanded program for immunization in Kenya. Human group A RV was detected in $37.8 \%$ of children hospitalized with acute gastroenteritis. This prevalence is within the range $(6-56 \%)$ previously detected in Kenya ${ }^{15,22}$ and higher than the previous study which was carried out in the same region. ${ }^{21}$ The detection rate of $\mathrm{RV}$ in this rural setting is similar to that previously reported in an urban setting in $2010 .{ }^{15}$

G9 genotype was the most common genotype, followed by G1 and then G8 which is uncommon globally, but detected in consistently in African countries. ${ }^{15}$ The globally common genotype G2 was detected at lower levels in this study. This trend of G2 has also been documented in a study from 2002 to 2004 in the coastal region of Kenya $^{24}$ and in another study that was done during 2000-2002 where G2 strains were detected in about $12 \%$ of samples. ${ }^{30}$ Over the last 8 years, no G2 has been documented in Kenya. The prevalence of G9 genotype was higher than that reported in the urban hospital setting in Kenya ( $50.9 \%$ vs. $8 \%$ ), while the prevalence of the G8 genotype was slightly lower (12.1\% vs. $20 \%)$ than that reported in the same urban setting. ${ }^{5}$ In addition, mixed infections and nontypeable samples were substantially lower in the eastern region compared with previous studies (mixed infection with $\mathrm{G}$ type, $1.3 \%$ vs. $8 \%$ and $\mathrm{G}$ nontypeable 5.1 $\%$ vs. $28 \%$ ). ${ }^{15,21}$ Genotype G9 has been dominant over most periods in the eastern region. ${ }^{21}$ Genotype G8 is increasingly becoming 1 of the dominant genotypes and genotype G1 had shown an increase in this region of Kenya over the last 2 years. Conversely, genotype G3 and G4 that were dominant in the 1990s were not detected in this study. ${ }^{22}$

Recently, G12 genotypes have been increasingly detected in various countries, including in Africa, ${ }^{31,32}$ but have not previously been reported from Kenya. ${ }^{15}$ G12 genotypes were confirmed in 3.1\% of samples by sequence analysis in the current study (data not shown).

Continuous monitoring of circulating genotypes in Kenya is important, because the frequency of the genotypes could change over time. Seasonal shifts of RV strains in a given geographical region have been observed in many countries, ${ }^{33-36}$ and it is a possible mechanism that the virus can use to evade herd immunity and ultimately persist in that human population. ${ }^{37}$ Notably, this was described for G2P [4] strain in South Africa. ${ }^{38}$ The diversity of genotypes as found in this study may be explained by the active interaction of people in the region, poor environmental conditions and close contact with domesticated animals.

Despite the substantial strain heterogeneity detected in this study, RV vaccines have been shown to provide heterotypic protection against a wide range of circulating strains. For example, in the Rotarix clinical trials in Africa, the vaccine, which is based on an attenuated G1P[8] human RV strain, provided similar protection against G1 strains (64\%) and non-G1 strains $(60 \%) .{ }^{8,11}$ However, further study on strain-specific vaccine effectiveness in settings like Kenya is still needed to monitor the impact of vaccines on the long-term epidemiology of strains in circulation. Continued monitoring of RV genotype distribution will be valuable to document the diversity and changes in the circulating strains that may be more important than ever following the introduction of RV vaccination

Our study had some limitations; for instance, the data collected were from one regional in the eastern Kenya and may not generally represent the actual burden of the disease and RV strains circulating in the entire country or even the eastern region. However, this study builds on the data generated in previous surveillance studies from this region using the same methodology and allows a temporal trend of circulating strains to be examined. The other limitation was that only a proportion of the RV-positive samples (157 of 189) were genotyped, although they were a representative of samples collected during the study period.

In conclusion, the present study confirms the current burden of RV gastroenteritis in younger children and also demonstrated a high prevalence of G9, G1 and G8 strains and the absence of 
G3 and G4 in Kenya during the study period 2009-2011. From our knowledge, this is the first time the G12 strain is being identified in Kenya. Due to the recent emergence of G12 RV worldwide, the findings from this study are important because they provide new information concerning the local and global spread of RV genotypes. Thus, continued monitoring and strain surveillance are important to document vaccine impact and potential emergence of new strains in the Kenyan community after vaccine introduction.

\section{ACKNOWLEDGMENTS}

This study was supported by a research grant from Investigator-Initiated Studies Program of Merck \& Co., Inc., NJ and partial support from the Institute of Primate Research. The authors acknowledge the technical assistance provided by Erick Omolo and Mary Galo of the Institute of Primate Research. The authors give special thanks to the surveillance team at Maua Methodist Hospital (Grace Safari, Lilian Mbirithu, Jeremy Marete, Mwathe Mutara, Florence Gitonga, Ken Maore and Samuel Kithinji) and Meru General Hospital (Mercy Kinyua, Monica Gitonga, Ephatus Karani, John Njeru and Cyprian Miriti) for helping in recruiting of participants for this study, N. M. K. acknowledges the small grant program support from the International Society for Infectious Diseases.

\section{REFERENCES}

1. Tate JE, Burton AH, Boschi-Pinto C, et al.; WHO-coordinated Global Rotavirus Surveillance Network. 2008 estimate of worldwide rotavirusassociated mortality in children younger than 5 years before the introduction of universal rotavirus vaccination programmes: a systematic review and meta-analysis. Lancet Infect Dis. 2012;12:136-141.

2. Sanchez-Padilla E, Grais RF, Guerin PJ, et al. Burden of disease and circulating serotypes of rotavirus infection in sub-Saharan Africa: systematic review and meta-analysis. Lancet Infect Dis. 2009;9:567-576.

3. Parashar U, Burton A, Lanata C, et al. Global mortality associated with rotavirus disease among children in 2004. J Infect Dis. 2009;200 (suppl 1):S9-15.

4. Ruiz-Palacios GM, Pérez-Schael I, Velázquez FR, et al.; Human Rotavirus Vaccine Study Group. Safety and efficacy of an attenuated vaccine against severe rotavirus gastroenteritis. $N$ Engl J Med. 2006;354:11-22.

5. Vesikari T, Matson DO, Dennehy P, et al.; Rotavirus Efficacy and Safety Trial (REST) Study Team. Safety and efficacy of a pentavalent human-bovine (WC3) reassortant rotavirus vaccine. $N$ Engl J Med. 2006;354:23-33.

6. Vesikari T, Karvonen A, Prymula R, et al. Efficacy of human rotavirus vaccine against rotavirus gastroenteritis during the first 2 years of life in European infants: randomised, double-blind controlled study. Lancet. 2007;370:1757-1763

7. Armah GE, Sow SO, Breiman RF, et al. Efficacy of pentavalent rotavirus vaccine against severe rotavirus gastroenteritis in infants in developing countries in sub-Saharan Africa: a randomised, double-blind, placebo-controlled trial. Lancet. 2010;376:606-614.

8. Madhi SA, Cunliffe NA, Steele D, et al. Effect of human rotavirus vaccine on severe diarrhea in African infants. N Engl J Med. 2010;362:289-298.

9. Zaman K, Dang DA, Victor JC, et al. Efficacy of pentavalent rotavirus vaccine against severe rotavirus gastroenteritis in infants in developing countries in Asia: a randomised, double-blind, placebo-controlled trial. Lancet. 2010;376:615-623.

10. Salinas B, Pérez Schael I, Linhares AC, et al. Evaluation of safety, immunogenicity and efficacy of an attenuated rotavirus vaccine, RIX4414: A randomized, placebo-controlled trial in Latin American infants. Pediatr Infect Dis J. 2005;24:807-816.

11. Steele AD, Neuzil KM, Cunliffe NA, et al. Human rotavirus vaccine Rotarix $^{\mathrm{TM}}$ provides protection against diverse circulating rotavirus strains in African infants: a randomized controlled trial. BMC Infect Dis. 2012;12:213.

12. Richardson V, Hernandez-Pichardo J, Quintanar-Solares M, et al. Effect of rotavirus vaccination on death from childhood diarrhea in Mexico. $N$ Engl J Med. 2010;362:299-305.

13. Pawinski R, Debrus S, Delem A, et al. Rotarix in developing countries: paving the way for inclusion in national childhood immunization programs in Africa. J Infect Dis. 2010;202 (suppl):S80-S86.
14. de Oliveira LH, Danovaro-Holliday MC, Matus CR, et al. Rotavirus vaccine introduction in the Americas: progress and lessons learned. Expert Rev Vaccines. 2008;7:345-353.

15. Mwenda JM, Ntoto KM, Abebe A, et al. Burden and epidemiology of rotavirus diarrhea in selected African countries: preliminary results from the African Rotavirus Surveillance Network. J Infect Dis. 2010;202 (suppl):S5-S11.

16. World Health Organization. Rotavirus vaccines: an update. Wkly Epidemiol Rec. 2009;84:533-40.

17. Estes M, Kapikian A. Rotaviruses. In: Knipe DM, Howley PM, Griffin DE, et al., eds. Fields Virology. 5th ed.Vol. 2. Philadelphia: Wolters Kluwer Lippincott Williams and Wilkins; 2007:1917-1974.

18. Matthijnssens J, Miño S, Papp H, et al. Complete molecular genome analyses of equine rotavirus A strains from different continents reveal several novel genotypes and a largely conserved genotype constellation. J Gen Virol. 2012;93(pt 4):866-875.

19. Gentsch JR, Laird AR, Bielfelt B, et al. Serotype diversity and reassortment between human and animal rotavirus strains: implications for rotavirus vaccine programs. J Infect Dis. 2005;192 (suppl 1):S146-S159.

20. Santos N, Hoshino Y. Global distribution of rotavirus serotypes/genotypes and its implication for the development and implementation of an effective rotavirus vaccine. Rev Med Virol. 2005;15:29-56.

21. Kiulia NM, Peenze I, Dewar J, et al. Molecular characterisation of the rotavirus strains prevalent in Maua, Meru North, Kenya. East Afr Med J. $2006 ; 83: 360-365$

22. Kiulia NM, Kamenwa R, Irimu G, et al. The epidemiology of human rotavirus associated with diarrhoea in Kenyan children: a review. J Trop Pediatr. 2008;54:401-405

23. Kiulia NM, Nyaundi JK, Peenze I, et al. Rotavirus infections among HIVinfected children in Nairobi, Kenya. J Trop Pediatr. 2009;55:318-323.

24. Nokes DJ, Peenze I, Netshifhefhe L, et al. Rotavirus genetic diversity, disease association, and temporal change in hospitalized rural Kenyan children. J Infect Dis. 2010;202 (suppl):S180-S186.

25. World Health Organization. Generic Protocols for (I) Hospital-based Surveillance to Estimate the Burden of Rotavirus Gastroenteritis in Children and (II) a Community-based Survey on Utilization of Health Care Services for Gastroenteritis in children. Field Test Version. WHO/V\&B/02.15. Geneva: World Health Organization; 2002.

26. Gouvea V, Glass RI, Woods P, et al. Polymerase chain reaction amplification and typing of rotavirus nucleic acid from stool specimens. J Clin Microbiol. 1990;28:276-282

27. Gentsch JR, Glass RI, Woods P, et al. Identification of group A rotavirus gene 4 types by polymerase chain reaction. J Clin Microbiol. 1992;30:1365-1373.

28. Iturriza-Gómara M, Isherwood B, Desselberger U, et al. Reassortment in vivo: driving force for diversity of human rotavirus strains isolated in the United Kingdom between 1995 and 1999. JVirol. 2001;75:3696-3705.

29. Iturriza-Gómara M, Kang G, Gray J. Rotavirus genotyping: keeping up with an evolving population of human rotaviruses. J Clin Virol. 2004;31:259-265.

30. Nyangao J, Page N, Esona M, et al. Characterization of human rotavirus strains from children with diarrhea in Nairobi and Kisumu, Kenya, between 2000 and 2002. J Infect Dis. 2010;202 (suppl):S187-S192.

31. Page NA, de Beer MC, Seheri LM, et al. The detection and molecular characterization of human G12 genotypes in South Africa. $J$ Med Virol. 2009;81:106-113

32. Matthijnssens J, Heylen E, Zeller M, et al. Phylodynamic analyses of rotavirus genotypes G9 and G12 underscore their potential for swift global spread. Mol Biol Evol. 2010;27:2431-2436.

33. Rahman M, Matthijnssens J, Goegebuer T, et al. Predominance of rotavirus G9 genotype in children hospitalized for rotavirus gastroenteritis in Belgium during 1999-2003. J Clin Virol. 2005;33:1-6.

34. Rahman M, Sultana R, Ahmed G, et al. Prevalence of G2P[4] and G12P[6] rotavirus, Bangladesh. Emerg Infect Dis. 2007;13:18-24.

35. Kirkwood CD, Bonifacea K, Bogdanovic-Sakran N, et al. Rotavirus strain surveillance - an Australian perspective of strains causing disease in hospitalised children from 1997 to 2007. Vaccine. 2009;27 (suppl 5):F102-F107.

36. Xu J, Yang Y, Sun J, et al. Molecular epidemiology of rotavirus infections among children hospitalized for acute gastroenteritis in Shanghai, China, 2001 through 2005. J Clin Virol. 2009;44:58-61.

37. Parra GI. Seasonal shifts of group A rotavirus strains as a possible mechanism of persistence in the human population. J Med Virol. 2009;81:568-571.

38. Page NA, Steele AD. Antigenic and genetic characterization of serotype G2 human rotavirus strains from the African continent. J Clin Microbiol. 2004;42:595-600. 